\title{
Impact of Preoperative Aspirin on Long-Term Outcomes in Diabetic Patients Following Coronary Artery Bypass Grafting: a Propensity Score Matched Study
}

Sleiman Sebastian Aboul-Hassan', MD, PhD; Tomasz Stankowski², MD, PhD; Jakub Marczak ${ }^{3}$ MD, PhD; Maciej Peksa ${ }^{1}$, MD; Marcin Nawotka ${ }^{1}$, MD; Ryszard Stanislawski ${ }^{1}$, MD; Lukasz Moskal ${ }^{4}$, MS; Adam Lipowski ${ }^{5}$, MD; Michel Pompeu B. O. Sá6, MD, MSc, PhD; Romuald Cichon ${ }^{1,7}$, MD, PhD

DOI: $10.21470 / 1678-9741-2020-0313$

\section{Abstract}

Introduction: This study aimed to determine the effect of preoperative aspirin administration on early and long-term clinical outcomes in patients suffering from diabetes mellitus (DM) undergoing coronary artery bypass grafting (CABG).

Methods: In this observational study, a total of 315 patients were included and grouped according to the time interval between their last aspirin dose and the time of surgery; patients who had been continued aspirin intake with last administered dose $\leq$ 24-hours before CABG $(n=144)$ and those who had been given the last dose of aspirin between 24 to 48 hours before CABG $(n=171)$.

Results: Multivariable analysis showed that the continuation of preoperative aspirin intake $\leq \mathbf{2 4}$ hours before CABG in patients with DM is associated with reduced incidence of 30-day major adverse cardiac and cerebral events (MACCE) $(P=0.004)$ as well as reduced incidence of composite 30-day mortality/MACCE
$(P=0.012)$. During mean follow-up of $37 \pm 17.5$ months, the unadjusted hazard ratio (HR) showed that aspirin $\leq \mathbf{2 4}$ hours prior CABG in patients with DM significantly reduced the incidence of MACCE and composite of mortality/MACCE during follow-up (HR: $0.50 ; 95 \%$ confidence interval [CI]: $0.29-0.87 ; P=0.014$ and HR: $0.61 ; 95 \% \mathrm{Cl}$ : 0.38-0.97; $P=0.039$, respectively). However, after propensity score (PS) matching, the PS-adjusted HR showed a non-significant trend towards the reduction of MACCE during follow-up (HR: 0.58; 95\% Cl: 0.31-1.06; $P=0.081$ ).

Conclusion: Continuation of preoperative aspirin intake $\leq 24$ hours before CABG in patients with DM is associated with reduced incidence of early MACCE, but without significant influence on long-term outcomes.

Keywords: Coronary Artery Bypass. Diabetes Mellitus. Aspirin. Incidence. Propensity Score.

\section{Abbreviations, acronyms \& symbols}

\begin{tabular}{llll}
\hline BMI & Body mass index & NYHA & $=$ New York Heart Association \\
CABG & = Coronary artery bypass grafting & ONCABG & $=$ On-pump coronary artery bypass grafting \\
CAD & C Coronary artery disease & OPCABG & $=$ Off-pump coronary artery bypass grafting \\
CAEs & $=$ Cerebral adverse events & OR & $=$ Odds ratio \\
CCB & $=$ Calcium channel blockers & PCI & $=$ Percutaneous coronary intervention \\
CLD & $=$ Chronic lung disease & PRBC & $=$ Packed red blood cells \\
CI & $=$ Confidence interval & PS & $=$ Propensity score \\
DM & $=$ Diabetes mellitus & PVD & $=$ Peripheral vascular disease \\
HR & $=$ Hazard ratio & RAASI & $=$ Renin angiotensin aldosterone inhibitors \\
LVEF & $=$ Left ventricular ejection fraction & RCT & $=$ Randomized controlled trial \\
MACCE & $=$ Major adverse cardiac and cerebral events & SD & $=$ Standard deviation \\
MI & $=$ Myocardial infarction & SMD & $=$ Standardized mean difference
\end{tabular}

1Department of Cardiac Surgery, Medinet Heart Center Ltd, Nowa Sol, Poland. ${ }^{2}$ Department of Cardiac Surgery, Sana-Heart Center Cottbus, Cottbus, Germany. ${ }^{3}$ Department of Cardiac Surgery, Trent Cardiac Centre, Nottingham University Hospital, Nottingham, United Kingdom.

${ }^{4}$ Department of Cardiac Surgery, Medinet Heart Center Ltd, Wroclaw, Poland. ${ }^{5}$ Department of Vascular Surgery, Nowa Sol Multidisciplinary Hospital, Nowa Sol, Poland. ${ }^{6}$ Division of Cardiovascular Surgery of Pronto Socorro Cardiológico de Pernambuco, PROCAPE, Universidade de Pernambuco, Recife, Pernambuco, Brazil.

${ }^{7}$ Department of Cardiac Surgery, Warsaw Medical University, Warsaw, Poland.
This study was carried out at the Department of Cardiac Surgery, Medinet Heart Center Ltd, Nowa Sol, Poland.

Correspondence Address:

Sleiman Sebastian Aboul-Hassan

(iD) https://orcid.org/0000-0003-4544-7466

Medinet Heart Center Ltd., Chalubinskiego 7 street, Nowa Sol, Poland - Zip Code: 67-100 E-mail: s.aboulhassan@gmail.com 


\section{INTRODUCTION}

Diabetes mellitus (DM) constitutes one of the major risk factors for developing cardiovascular diseases, most importantly, coronary artery disease $(C A D)^{[1]}$. Patients burdened with $\mathrm{DM}$ are at increased risk of adverse cardiovascular events by two-fold to three-fold when compared to patients without $\mathrm{DM}^{[2]}$. Recent guidelines of the European Society of Cardiology/European Association for Cardio-Thoracic Surgery, or ESC/EACTS, on myocardial revascularization recommended coronary artery bypass grafting $(C A B G)$ as the method of choice in the treatment of patients with multivessel CAD and DM (Class I-a) ${ }^{[3]}$. Although CABG is the most effective revascularization therapy in these patients, a recent meta-analysis showed that pre-existing DM places patients at higher risk for inferior long-term postoperative outcomes ${ }^{[4]}$. This observation could be explained by several possible mechanisms including insulin resistance, release of fatty acids in addition to other metabolic phenomena that may in turn lead to a series of events such as endothelial dysfunction, oxidative stress, inflammation, and platelet hyper-reactivity, which affects the vascular wall and enhances the progress of atherosclerosis $s^{[5]}$. It is a well-established fact that aspirin due to its antiplatelet and anti-inflammatory function is beneficial for patients with CAD. The administration of aspirin within 48 hours following CABG is associated with a reduced incidence of major adverse cardiac and cerebral events (MACCE) $)^{[6]}$. Despite aspirin's beneficial effect in patients following $C A B G$, its continuation throughout the perioperative period or the time of its discontinuation prior to surgery remain ambiguous.

Current guidelines differ in their recommendations regarding the use of preoperative aspirin before CABG. While some recommend its continuation until the day of surgery ${ }^{[7-8]}$, others recommend its discontinuation at least a few days prior elective CABG to decrease the risk of bleeding ${ }^{[9]}$. On the other hand, a recent meta-analysis showed that the continuation of preoperative aspirin intake is associated with reduced early mortality as well as reduced incidence of perioperative myocardial infarction (MI) following cardiac surgery ${ }^{[10]}$. Studies have shown that platelet function gradually recovers within 72 hours reaching full recovery within 96 hours after aspirin cessation $^{[11-12]}$. Therefore, withdrawal of aspirin a few days before CABG could potentially lead to a partial or complete recovery of platelet function which could in turn increase the risk of thrombo-occlusive events in patients with DM. Recent studies showed that the continuation of aspirin intake with its last dose within 24 hours before CABG is associated with improved postoperative outcomes in terms of mortality, MACCE, and acute kidney injury ${ }^{[13-15]}$. Despite abundant evidence generated by the recent studies or meta-analyses, none of them evaluated the impact of preoperative aspirin on postoperative outcomes following CABG in patients with DM. Therefore, we aimed to investigate the effect of preoperative aspirin administration on early and long-term clinical outcomes in patients suffering from $D M$ and $C A D$ undergoing $C A B G$.

\section{METHODS}

This observational, retrospective study was designed according to the Strengthening the Reporting of Observational Studies in Epidemiology, or STROBE, guidelines ${ }^{[16]}$. Between
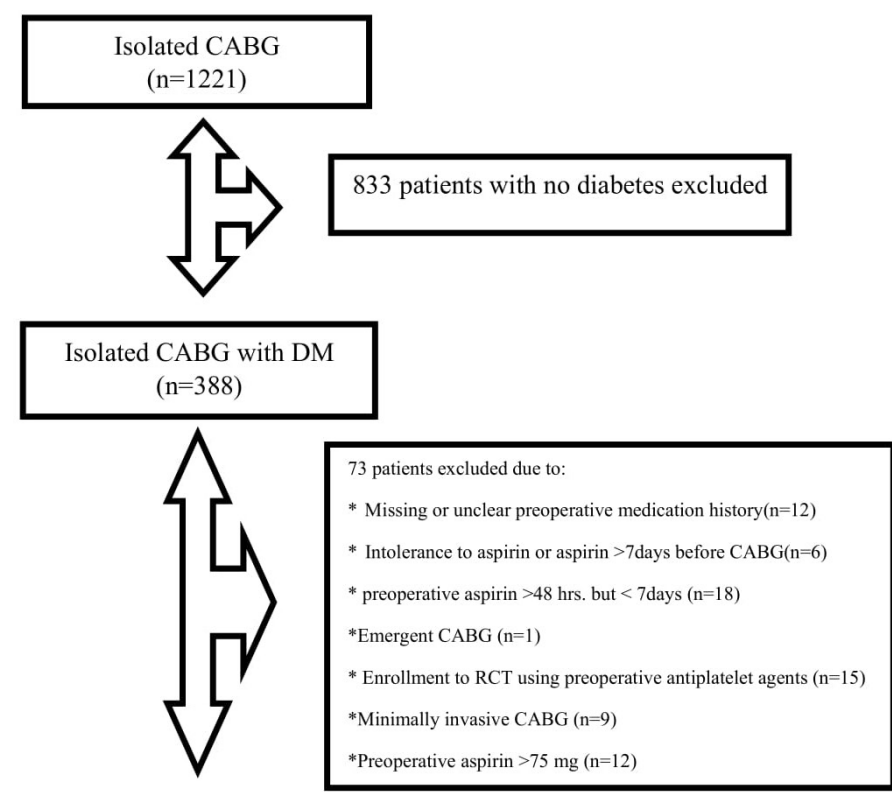

Isolated $\mathrm{CABG}$ with $\mathrm{DM}$ $(n=315)$

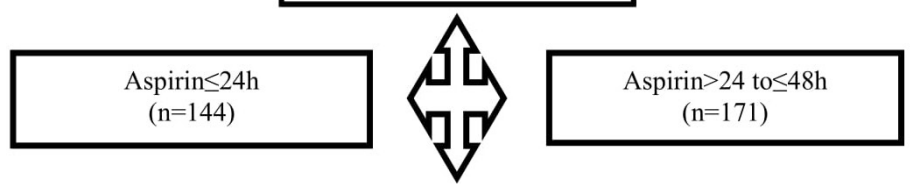

Propensity score matching

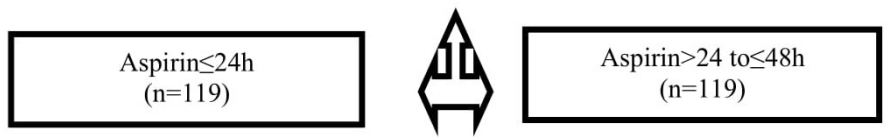

Fig. 1 - Patients' flowchart diagram. $C A B G=$ coronary artery bypass grafting; $D M=$ diabetes mellitus; $R C T=$ randomized controlled trial 
January 2014 and April 2018, 1221 patients underwent isolated CABG at the Department of Cardiac Surgery, Medinet Heart Center Ltd, Nowa Sol, Poland. Of these, 388 patients were diagnosed with type $2 \mathrm{DM}$. After analyzing and reviewing the data, 315 patients were included in the study and were divided into groups according to the time interval between their last aspirin dose and the time of surgery. Patients who had been continued aspirin with last administered dose $\leq 24$ hours before CABG $(n=144)$ and those who had been given the last dose of aspirin between 24 to 48 hours before CABG $(n=171)$. Seventy-three patients were excluded due to: missing or unclear preoperative medication history $(n=12)$, aspirin administration for more than 48 hours before CABG but less than seven days $(n=18)$, documented intolerance to aspirin or did not take aspirin for more than seven days $(n=6)$, emergency CABG $(n=1)$, minimally invasive $C A B G(n=9)$, enrollment to ongoing randomized controlled trial $(\mathrm{RCT})$ testing antiplatelet therapy following CABG $(n=15)$, and administration of $>75 \mathrm{mg}$ of aspirin before CABG $(n=12)$. Figure 1 represents the patients' flowchart diagram. The study was approved by the institutional review board at Medinet Heart Center and by the Bioethics Committee of Wroclaw's Medical University, Poland. An individual consent of the patient for anonymous data analysis was waived by the Committee.

Patients' demographics, clinical characteristics, medications, and postoperative outcomes were retrieved from the database of Medinet Heart Center, Nowa Sol, Poland. Preoperative aspirin dosing as well as the time of its last administration were both obtained from patients' medication chart. The data covering preoperative dosage and time of the last aspirin dose taken by patients admitted to the hospital the day before surgery were obtained from the preoperative medication questionnaire. As for patients transferred from other departments, preoperative aspirin dosage and timing were obtained from patients' discharge letter. Postoperatively, all patients received aspirin between six and 24 hours following CABG and that was continued daily thereafter. Follow-up data were retrieved from the national health care registry of the Ministry of Health of the Republic of Poland, that stores and analyzes all health-related data.

Study endpoints included early (30-day) and long-term MACCE and the composite of mortality and MACCE. Safety endpoints included postoperative chest tube drainage at 24 hours, postoperative transfusion rate of packed red blood cells (pRBC), need for re-exploration of the chest for bleeding, and duration of hospital stay. MACCE was defined as the occurrence of MI or cerebral adverse events (CAEs). MI was defined according to the third international definition of $M{ }^{[17]}$. CAEs were defined as the development of a new permanent or transient (lasting up to 72 hours) neurologic deficit as confirmed by stroke team member assessment of the patient and computed tomography of the central nervous system, magnetic resonance imaging, or at autopsy examination.

Statistical analyses were performed using statistical software STATISTICA (TIBCO Software Inc. 2017, data analysis software system, version-13, Palo Alto, United States of America). Continuous variables were expressed as means \pm standard deviation (SD), while categorical variables as number and percentages. For continuous data, Student's t-test or MannWhitney's U-test was used for between groups comparisons, while categorical variables were compared with Pearson's $x^{2}$ test.

To identify independent predictors of 30-day MACCE and composite of 30-day mortality and MACCE, we built a multivariable logistic regression model for the whole cohort by using all preoperative variables presented in Table 1. In addition to the above, operative and postoperative outcomes such as the type of surgery, number of grafts performed, duration of surgery, inotropic support, total arterial revascularization, and pRBC transfusion were included into the model. Multivariable logistic regression analysis was performed using stepwise backward regression. Only relevant covariates identified during univariate analysis with a $P$-value $\leq 0.1$ were included in the final computation of a multivariable logistic model.

To reduce the risk of selection bias inherent to retrospective, observational studies, a propensity score (PS) matching was used to match patients between the groups. PS were generated from a multivariable logistic regression model based on seven preoperative variables: age, gender, left ventricular ejection fraction, recent $\mathrm{Ml}(<90$ days), renal insufficiency, and preoperative medication $\leq 24$ hours (B-blockers and statins). Patients were then matched in 1:1 fashion using caliper matching method without replacement with a caliper width of 0.2 SD of the logit of PS ${ }^{[18-19]}$. The balance of the covariates was tested using standardized mean difference (SMD). Statistical guidelines suggest a meaningful covariate balance of the variables used to generate the PS between the two groups to be between - 0.1 $<\mathrm{SMD}<0.1^{[18]}$. Matched data were analyzed using procedures for matched analyses. For continuous data, Wilcoxon matchedpairs test was used, while McNemar's test was used for binary outcomes.

Freedom from MACCE as well as freedom from composite mortality and MACCE at follow-up in the unadjusted and PSadjusted populations were estimated using the Kaplan-Meier method and were expressed as percentages. Log-rank test was used to compare the data. Hazard ratio (HR) in both populations with 95\% confidence interval (Cl) was derived from the Cox proportional hazards model. Statistical significance was defined as a $P$-value $<0.05$.

\section{RESULTS}

Baseline and operative characteristics of the unadjusted and PS-adjusted populations are presented in Tables 1 and 2. In the unadjusted study groups, female patients were more likely to be found in the group where aspirin was discontinued between 24 and 48 hours before CABG, whereas patients in whom aspirin has been continued $\leq 24$ hours before $C A B G$ were more likely to receive concurrent preoperative medication such as B-blockers and statins. There were no significant differences between both groups in terms of operative characteristics such as type of surgery, duration of surgery, number of grafts performed, and number of patients with total arterial revascularization (Table 2). After performing PS matching, the differences in baseline characteristics between both groups were eliminated (Table 1). PS matching selected 119 matched pairs for final comparison. 
Table 1. Baseline characteristics of patients with diabetes undergoing coronary artery bypass grafting grouped according to time interval of aspirin discontinuation.

\begin{tabular}{|c|c|c|c|c|c|c|c|}
\hline & \multicolumn{3}{|c|}{ Before matching } & \multicolumn{4}{|c|}{ After matching } \\
\hline & \multicolumn{2}{|c|}{ Aspirin } & \multirow{3}{*}{$P$-value } & \multicolumn{2}{|c|}{ Aspirin } & \multirow{3}{*}{$P$-value } & \multirow{3}{*}{ SMD } \\
\hline & $\leq 24 \mathrm{~h}$ & $>24$ to $\leq 48 h$ & & $\leq 24 \mathrm{~h}$ & $>24$ to $\leq 48 h$ & & \\
\hline & $(n=144)$ & $(n=171)$ & & $(n=119)$ & $(n=119)$ & & \\
\hline Age (years) & $66.3 \pm 7.63$ & $65.5 \pm 7.33$ & 0.35 & $65.6 \pm 7.43$ & $65.6 \pm 7.55$ & 1 & 0.000 \\
\hline Female gender & $37(25.6)$ & $62(36.2)$ & 0.045 & $36(30.2)$ & $34(28.5)$ & 0.77 & 0.044 \\
\hline PVD & $34(23.6)$ & $39(22.8)$ & 0.86 & $27(22.6)$ & $29(24.3)$ & 0.75 & \\
\hline $\mathrm{BMI}\left(\mathrm{kg} / \mathrm{m}^{2}\right)$ & $30.1 \pm 4.81$ & $30.3 \pm 4.37$ & 0.70 & $29.9 \pm 4.89$ & $30.2 \pm 4.21$ & 0.61 & \\
\hline CLD & $12(8.3)$ & $13(7.6)$ & 0.81 & $10(8.4)$ & $6(5.0)$ & 0.30 & \\
\hline LVEF (\%) & $48.7 \pm 9.50$ & $49.6 \pm 10.29$ & 0.42 & $49.3 \pm 9.19$ & $49.1 \pm 10.2$ & 0.87 & 0.020 \\
\hline History of CAEs & $18(12.5)$ & $25(14.6)$ & 0.58 & $16(13.4)$ & $20(16.8)$ & 0.47 & \\
\hline Diabetes on insulin & $79(54.8)$ & $91(53.2)$ & 0.77 & $61(51.2)$ & $67(56.3)$ & 0.43 & \\
\hline NYHA III-IV & $18(12.5)$ & $14(8.1)$ & 0.20 & $16(13.4)$ & $10(8.4)$ & 0.21 & \\
\hline Recent MI (<90 days) & $48(33.3)$ & $62(36)$. & 0.58 & $40(33.6)$ & $38(31.9)$ & 0.78 & 0.042 \\
\hline Urgent & $86(59.7)$ & $92(53.8)$ & 0.29 & $72(60.5)$ & $64(53.7)$ & 0.29 & \\
\hline Previous PCl & $27(18.7)$ & $45(26.3)$ & 0.11 & $23(19.3)$ & $33(27.7)$ & 0.12 & \\
\hline Hypertension & $137(95.1)$ & $163(95.3)$ & 0.93 & $113(94.9)$ & $114(95.7)$ & 0.75 & \\
\hline Hyperlipidemia & $95(65.9)$ & $110(64.3)$ & 0.76 & $76(63.8)$ & $75(63.0)$ & 0.89 & \\
\hline Active smoker & $37(25.6)$ & $42(24.5)$ & 0.81 & $31(26.0)$ & $28(23.5)$ & 0.65 & \\
\hline $\mathrm{N}$ of diseased vessels & $2.98 \pm 0.98$ & $2.92 \pm 0.92$ & 0.58 & $2.94 \pm 0.96$ & $2.91 \pm 0.93$ & 0.81 & \\
\hline Renal insufficiency* & $79(54.8)$ & $101(59.0)$ & 0.45 & $67(56.3)$ & $67(56.3)$ & 1 & 0.000 \\
\hline
\end{tabular}

Preoperative medications $\leq 24$ hours

\begin{tabular}{|c|c|c|c|c|c|c|c|}
\hline B-blockers & $101(70.1)$ & $92(53.8)$ & 0.003 & $76(63.8)$ & $75(63.0)$ & 0.89 & 0.019 \\
\hline$C C B$ & $11(7.6)$ & $13(7.6)$ & 0.99 & $8(6.7)$ & $9(7.5)$ & 0.80 & \\
\hline RAAS & $40(27.7)$ & $43(25.1)$ & 0.59 & $32(26.8)$ & $36(30.2)$ & 0.56 & \\
\hline Statins & $113(78.4)$ & $104(60.8)$ & 0.0009 & 88 (73.9) & 85 (71.4) & 0.66 & 0.07 \\
\hline Clopidogrel $<7$ days & $14(9.7)$ & $26(15.2)$ & 0.14 & $12(10.0)$ & $19(15.9)$ & 0.18 & \\
\hline
\end{tabular}

Data are expressed as mean \pm standard deviation or $\mathrm{n}(\%)$, unless otherwise indicated.

*Renal insufficiency is defined as estimated glomerular filtration rate $<85 \mathrm{ml} / \mathrm{min} / 1.73 \mathrm{~m}^{2}$.

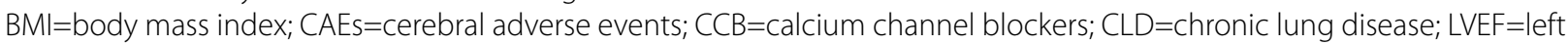
ventricular ejection fraction; $\mathrm{Ml}=$ myocardial infarction; NYHA=New York Heart Association; $\mathrm{PCl}=$ percutaneous coronary intervention; $\mathrm{PVD}=$ peripheral vascular disease; RAASI=renin angiotensin aldosterone inhibitors; SMD=standardized mean difference

The two matched groups were balanced $(-0.1<\mathrm{SMD}<0.1)$ (Table 1). Mirrored histogram showed adequate PS overlapping (Figure 2).

Multivariable analysis in the entire cohort showed that continuation of preoperative aspirin with the last dose administered within 24 hours before CABG in patients with DM is independently associated with reduced incidence of 30-day MACCE (odds ratio [OR]: $0.14 ; 95 \% \mathrm{Cl}: 0.04-0.54 ; P=0.004$ ) as well as reduced incidence of composite 30-day mortality and MACCE (OR: 0.24; 95\% Cl: 0.08-0.73; P=0.012). Other significant predictors of 30-day MACCE were as follows: active smoking, use of inotropic support, and number of stenosed vessels. Age, inotropic support, and number of stenosed vessels were found to increase the probability of composite 30-day mortality and MACCE. After PS matching, patients with DM receiving aspirin within 24-hours prior CABG had a lower incidence of 
Table 2. Operative characteristics.

\begin{tabular}{|c|c|c|c|c|c|c|}
\hline \multirow{3}{*}{ Type of surgery } & \multicolumn{3}{|c|}{ Before matching } & \multicolumn{3}{|c|}{ After matching } \\
\hline & \multirow{2}{*}{$\begin{array}{c}\text { Aspirin } \leq \\
24 h \\
(n=144)\end{array}$} & \multirow{2}{*}{$\begin{array}{c}\text { Aspirin }>24 \\
\text { to } \leq 48 h \\
(n=171)\end{array}$} & \multirow[t]{2}{*}{$P$-value } & $\begin{array}{c}\text { Aspirin } \leq \\
24 \mathrm{~h}\end{array}$ & $\begin{array}{c}\text { Aspirin }>24 \\
\text { to } \leq 48 \mathrm{~h}\end{array}$ & \multirow[t]{2}{*}{$P$-value } \\
\hline & & & & $(n=119)$ & $(n=119)$ & \\
\hline OPCABG & $119(82.6)$ & $143(83.6)$ & 0.81 & $100(84.0)$ & $99(83.1)$ & 0.86 \\
\hline ONCABG & $25(17.4)$ & $28(16.4)$ & 0.81 & $19(16.0)$ & $20(16.9)$ & 0.86 \\
\hline Duration (min) & $168 \pm 53$ & $167 \pm 51$ & 0.86 & $169 \pm 53$ & $165 \pm 48$ & 0.48 \\
\hline Number of grafts & $2.51 \pm 0.83$ & $244 \pm 0.75$ & 0.43 & $2.51 \pm 0.84$ & $2.45 \pm 0.75$ & 0.50 \\
\hline Total arterial revascularization & $33(22.9)$ & $52(30.4)$ & 0.13 & $28(23.5)$ & $33(27.7)$ & 0.45 \\
\hline
\end{tabular}

Data are expressed as mean \pm standard deviation or $n(\%)$, unless otherwise indicated.

$\mathrm{ONCABG}=$ on-pump coronary artery bypass grafting; $O P C A B G=$ off-pump coronary artery bypass grafting

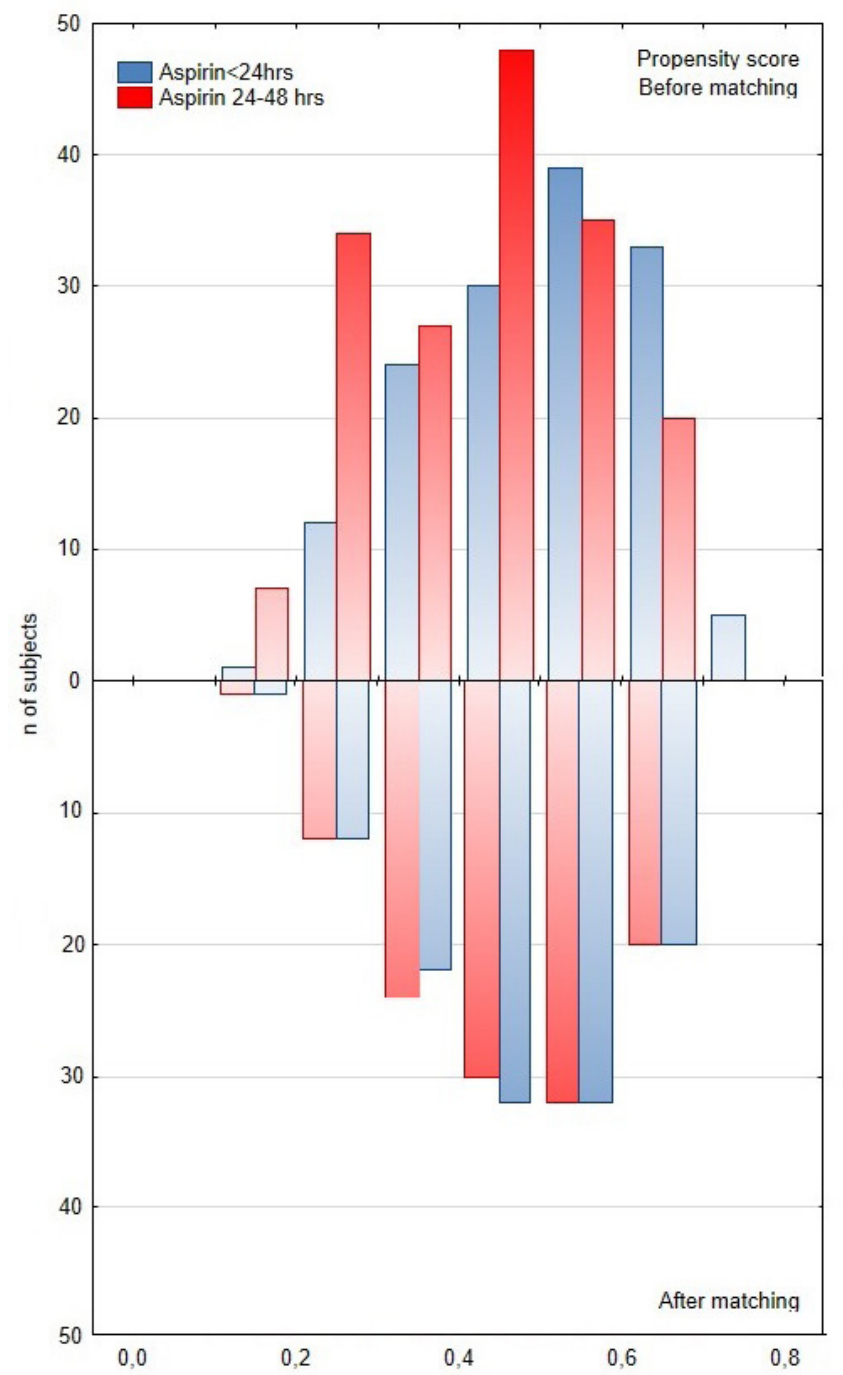

Fig. 2 -Mirrored histogram showing the propensity score distribution before and after matching in both groups. 30-day MACCE in comparison with patients who discontinued aspirin between 24 and 48 hours before CABG (3.3\% vs. 10.0\%, respectively, $P=0.04$ ) (Table 3 ). A non-significant trend towards reduced incidence of composite 30-day mortality and MACCE was observed in patients receiving aspirin $\leq 24$ hours prior to CABG (4.2\% vs. 10.9\%, $P=0.06$ ) (Table 3). Before as well as after the PS matching, no significant differences were observed in terms of reoperation for bleeding, chest tube drainage, $\mathrm{PRBC}$ transfusions, and duration of hospital stay (Table 3 ).

In the unmatched population, mean follow-up time was $37 \pm 17.5$ months. Freedom from MACCE at 12, 36, and 60 months were $93.1 \%, 87.0 \%$, and $82.5 \%$ in the aspirin $\leq 24$-hour group and $86.7 \%, 75.8 \%$, and $68.3 \%$ in the aspirin $>24$-to- $\leq 48$-hour group (log-rank $P=0.012$, Figure 3A). Freedom form composite mortality and MACCE at 12,36, and 60 months were $89.2 \%, 82.1 \%$, and $72.7 \%$ in the aspirin $\leq 24$-hour group and $83.1 \%, 72.7 \%$, and $59.6 \%$ in the aspirin $>24$-to- $\leq 48$-hour group ( $\log$ rank $P=0.037$, Figure $4 \mathrm{~A})$. In the PS-matched cohort, the mean follow-up time was $38 \pm 17$ months. Freedom from MACCE at 12, 36, and 60 months were $92.6 \%, 86.0 \%$, and $80.8 \%$ in the aspirin $\leq 24$-hour group and $87.7 \%, 77.3 \%$, and $69.4 \%$ in the aspirin $>24$-to- $\leq 48$-hour group (log rank $P=0.077$, Figure $3 B$ ). Whereas freedom from composite mortality and MACCE at 12, 36, and 60 months were 87.9\%, 80.4\%, and $68.9 \%$ in the aspirin $\leq 24$-hour group and $84.2 \%, 74.7 \%$, and $60.7 \%$ in the aspirin $>24$-to- $\leq 48$-hour group (log rank $P=0.26$, Figure 4B). MACCE as well as composite of mortality and MACCE were assessed using Cox regression analysis. The unadjusted HR showed that aspirin $\leq 24$ hours prior to CABG in patients with DM significantly reduced the incidence of MACCE and composite of mortality and MACCE during follow-up (HR: 0.50; 95\% Cl: 0.29-0.87; $P=0.014$ and HR: $0.61 ; 95 \% \mathrm{Cl}$ : 0.38-0.97; respectively; $P=0.039$ ). However, after PS matching, the PS-adjusted HR showed a nonsignificant trend towards reduced incidence of MACCE during follow-up (HR: 0.58; 95\% Cl: 0.31-1.06; P=0.081).

\section{DISCUSSION}

DM is one of the main risk factors for CAD and is known to be associated with an increased rate of cardiovascular 
Table 3. Early postoperative outcomes following coronary artery bypass grafting in diabetic patients.

\begin{tabular}{|c|c|c|c|c|c|c|}
\hline & \multicolumn{3}{|c|}{ Before matching } & \multicolumn{3}{|c|}{ After matching } \\
\hline 30-day mortality & $1(0.69)$ & $7(4.0)$ & 0.09 & $1(0.84)$ & $5(4.2)$ & 0.22 \\
\hline 30-day MACCE & $4(2.7)$ & $18(10.5)$ & 0.01 & $4(3.3)$ & $12(10.0)$ & 0.04 \\
\hline Inotropic support & $36(25)$ & $44(25.7)$ & 0.88 & $30(25.2)$ & $30(25.2)$ & 0.88 \\
\hline Reoperation for bleeding & $7(4.8)$ & $9(5.2)$ & 0.87 & $7(5.8)$ & $8(6.7)$ & 1 \\
\hline Chest tube drainage at $\mathrm{t}=24$ hours $(\mathrm{ml})$ & $646 \pm 314$ & $610 \pm 291$ & 0.29 & $635 \pm 316$ & $605 \pm 309$ & 0.31 \\
\hline pRBC transfusion & $68(47.2)$ & $73(42.6)$ & 0.42 & $57(47.8)$ & 45(37.8) & 0.13 \\
\hline
\end{tabular}

Data are expressed as mean \pm standard deviation or $n(\%)$, unless otherwise indicated.

${ }^{*}$ Chi-square test or $t$-test.

+McNemar test or Wilcoxon matched pairs test.

MACCE=major adverse cardiac and cerebral events; $\mathrm{pRBC}=$ packed red blood cell

events when compared to patients with normoglycemia ${ }^{[1-2]}$. Endothelial dysfunction caused by DM triggers inflammation, which is associated with cardiovascular events in these patients ${ }^{[20]}$. Hyperglycemia creates a disproportion between nitric oxide and reactive oxygen species as well as reactive nitrogen species resulting in endothelial dysfunction ${ }^{[21-22]}$. Therefore, DM leads to vascular wall damage which in addition to platelet hyperreactivity enhances the progress of atherosclerosis, which in turn may contribute to prothrombotic conditions ${ }^{[5]}$. On the other hand, studies have shown increased thromboembolic events in patients undergoing $C A B G$ due to the use of extracorporeal circulation. On-pump CABG is known to induce inflammation and promotes endothelial injury ${ }^{[23]}$. While, in patients undergoing offpump CABG, a hypercoagulable state and platelet hyper-reactivity are observed ${ }^{[24-26]}$. Patients with DM undergoing CABG could be, therefore, at even higher risk for thromboembolic events as this was recently proven by a meta-analysis showing that DM constitutes a risk factor for worse long-term outcomes after $\mathrm{CABG}^{[4]}$. Aspirin with its antiplatelet and anti-inflammatory effects could act as a preventive measure for early thromboembolic events following CABG, especially in patients with DM, in whom aspirin was shown to have a protective effect not only for secondary prevention but also for primary prevention of first cardiovascular events ${ }^{[27]}$. The pharmacological mechanism of aspirin action is well described. Duration of the antiplatelet effect is determined by cyclooxygenase re-synthesis, which in case of matured platelets, its full replacement is needed to recover cyclooxygenase. However, studies have shown that the function of the platelets could gradually recover within 72 hours after aspirin withdrawal and fully normalizing its function within 96 hours $^{[11-12]}$. Therefore, a partial recovery of the platelet function could be observed as soon as 24 to 48 hours after aspirin discontinuation.
A recent meta-analysis showed that the continuation of preoperative aspirin intake is associated with reduced early mortality as well as reduced incidence of perioperative $\mathrm{Ml}$ following cardiac surgery ${ }^{[10]}$. On the other hand, recent studies showed that the continuation of aspirin intake with the last aspirin dose administered within 24 hours before CABG is associated with reduced early mortality as well as reduced incidence of MACCE ${ }^{[13-14]}$. Deja et al. ${ }^{[28]}$ in a well-designed RCT showed that the administration of preoperative aspirin prior CABG is associated with a decreased long-term hazard of $\mathrm{MI}$ or repeated revascularization ( $\mathrm{HR}: 0.58$; $95 \% \mathrm{Cl}$ : 0.33-0.99; $\mathrm{P}=0.046$ ), whereas Xiao et al. ${ }^{[29]}$ observed a trend toward decreased midterm hazard of angina recurrence in patients in whom aspirin was continued till surgery. However, none of the available metaanalyses nor studies described the effect of preoperative aspirin in patients with DM undergoing CABG. Therefore, in this study, we aimed on determining the effect of preoperative aspirin use on early and long-term outcomes according to time intervals in patients with DM following CABG. We showed a clear benefit in patients with DM who took aspirin 24 hours or less prior CABG without increasing the risk of bleeding.

\section{Limitations}

The present study has several limitations. Firstly, this was a single-center, retrospective study without randomization. To reduce the risk of bias, we used PS matching. However, despite its use, many factors may still affect outcomes. Secondly, we were unable to adjust for antifibrinolytic agents due to a systematic lack of its reporting. Thirdly, we had no influence on postoperative medication at follow-up and this factor could not be adjusted in long-term follow-up. Finally, we are aware that 
- Preoperative Aspirin $24 \mathrm{hrs}$

- - Preoperative Aspirin 24-48 hrs

-.. Confidence limits of Aspirin 24-48 hrs group

... Confidence limits of Aspirin $<24 \mathrm{hrs}$ group

A

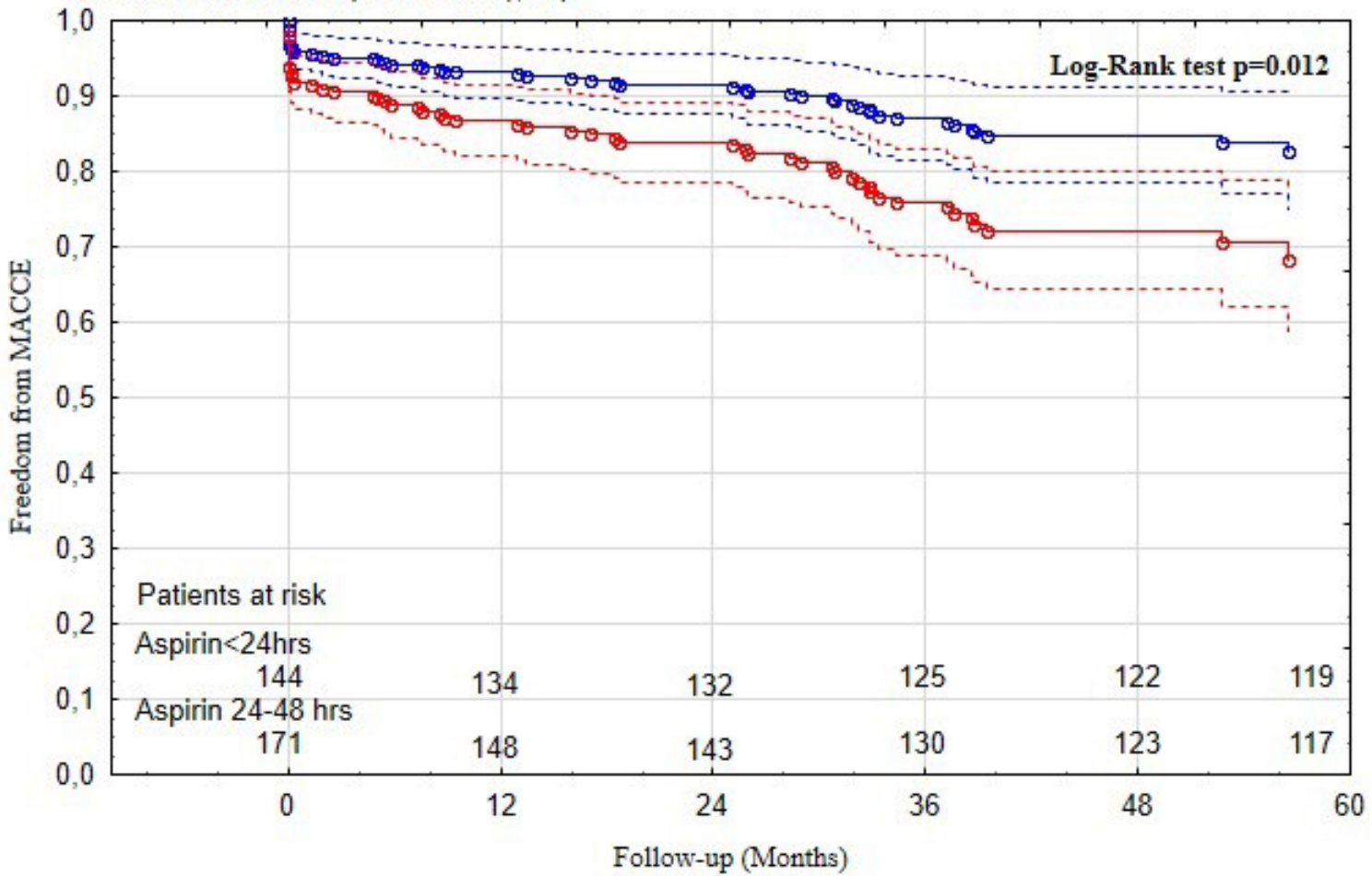

B

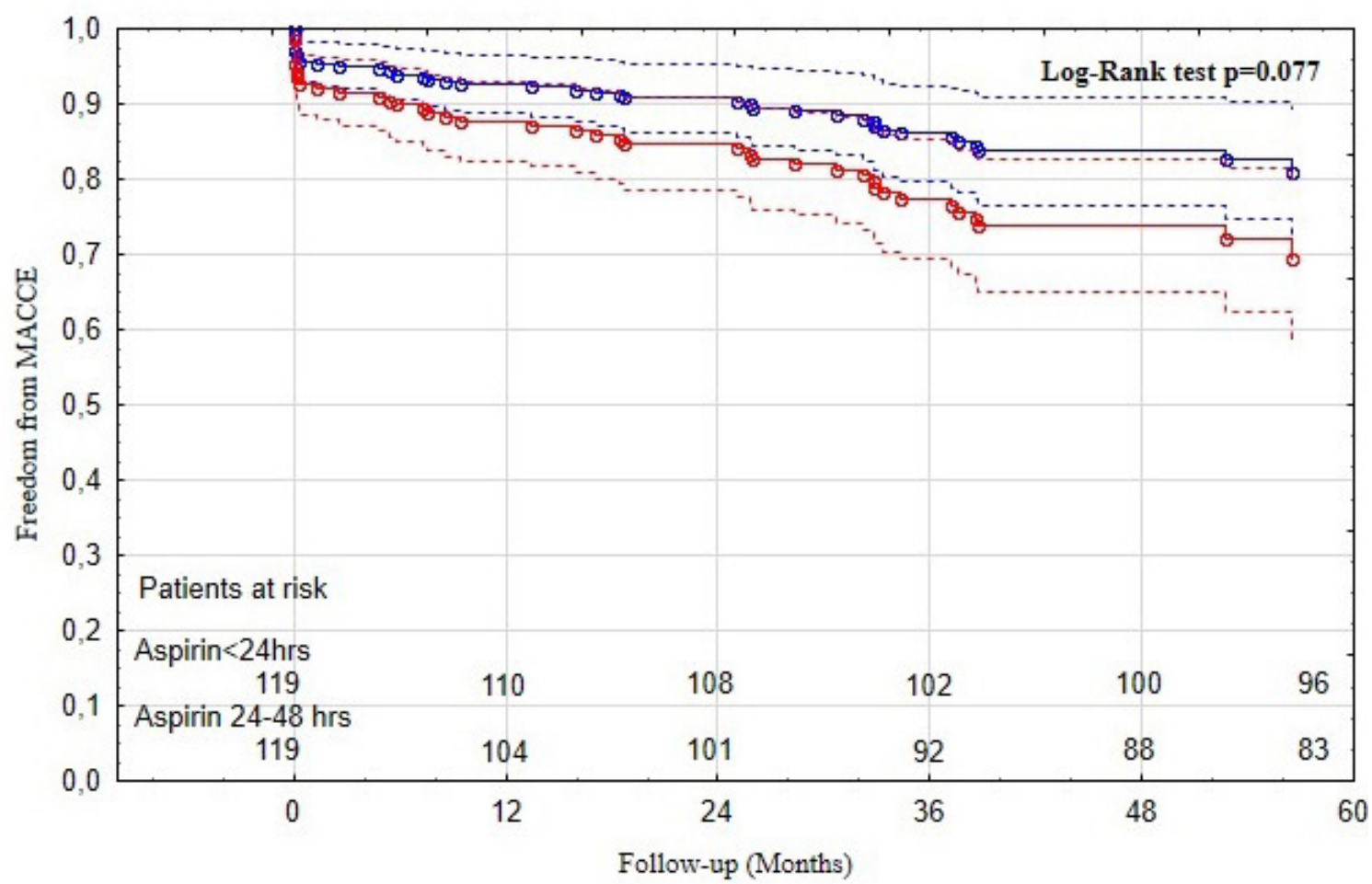

Fig. 3 - Kaplan-Meier freedom from major adverse cardiac and cerebral events (MACCE) curve. A) Before propensity score matching; B) after propensity score matching. 

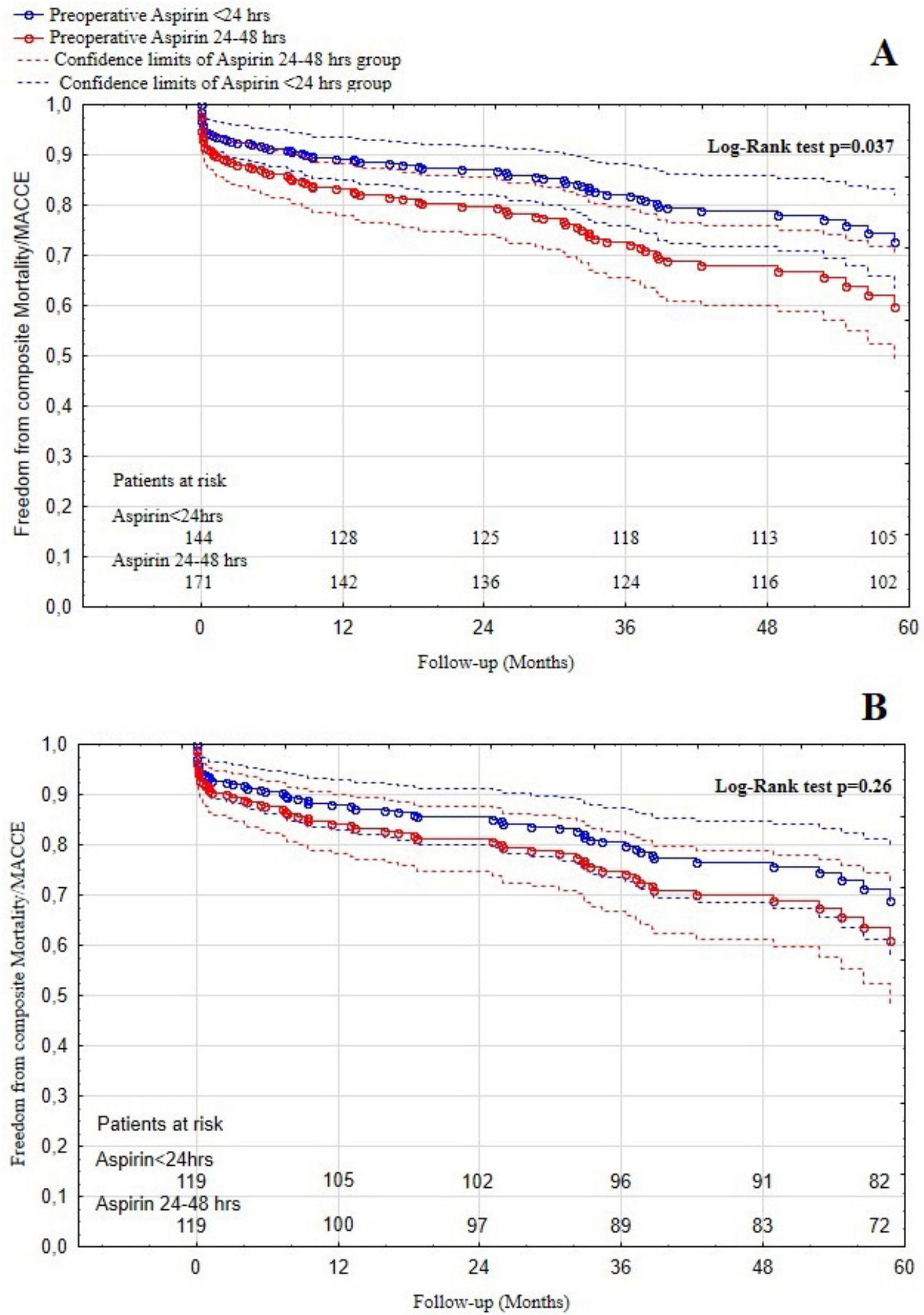

Fig. 4 - Kaplan-Meier freedom from composite mortality and major adverse cardiac and cerebral events (MACCE) curve. A) Before propensity score matching; B) after propensity score matching. 
this study might be underpowered by the sample size. Therefore, large multi-center, randomized controlled studies are required in patients with DM undergoing CABG.

\section{CONCLUSION}

In conclusion, in patients with DM undergoing CABG, continuation of preoperative aspirin intake till the day of surgery with the last dose administered within 24 hours or less prior CABG is associated with reduced incidence of early MACCE, but without significant influence on long-term outcomes.

\section{No financial support.}

No conflict of interest.

\section{Authors' roles \& responsibilities}

SSAH Substantial contributions to the conception or design of the work; or the acquisition, analysis, or interpretation of data for the work; drafting the work or revising it critically for important intellectual content; final approval of the version to be published

TS Drafting the work or revising it critically for important intellectual content; final approval of the version to be published

JM Interpretation of data for the work; drafting the work or revising it critically for important intellectual content; final approval of the version to be published

MP Drafting the work or revising it critically for important intellectual content; final approval of the version to be published

MN Drafting the work or revising it critically for important intellectual content; final approval of the version to be published

RS

Drafting the work or revising it critically for important intellectual content; final approval of the version to be published

LM Drafting the work or revising it critically for important intellectual content; final approval of the version to be published

AL Drafting the work or revising it critically for important intellectual content; final approval of the version to be published

MPBOS Drafting the work or revising it critically for important intellectual content; final approval of the version to be published

RC Drafting the work or revising it critically for important intellectual content; final approval of the version to be published

\section{REFERENCES}

1. Go AS, Mozaffarian D, Roger VL, Benjamin EJ, Berry JD, Borden WB, et al. Executive summary: heart disease and stroke statistics--2013 update: a report from the American heart association. Circulation. 2013;127(1):143-52. doi:10.1161/CIR.0b013e318282ab8f.
2. Emerging Risk Factors Collaboration, Sarwar N, Gao P, Seshasai SR, Gobin $R$, Kaptoge $S$, et al. Diabetes mellitus, fasting blood glucose concentration, and risk of vascular disease: a collaborative meta-analysis of 102 prospective studies. Lancet. 2010;375(9733):2215-22. Erratum in: Lancet. 2010;376(9745):958. Hillage, H L [corrected to Hillege, H L]. doi:10.1016/ S0140-6736(10)60484-9.

3. Neumann FJ, Sousa-Uva M, Ahlsson A, Alfonso F, Banning AP, Benedetto U, et al. 2018 ESC/EACTS guidelines on myocardial revascularization. Eur Heart J. 2019:40(2):87-165. Erratum in: Eur Heart J. 2019;40(37):3096. doi:10.1093/ eurheartj/ehy394.

4. Bundhun PK, Bhurtu A, Yuan J. Impact of type 2 diabetes mellitus on the long-term mortality in patients who were treated by coronary artery bypass surgery: a systematic review and meta-analysis. Medicine (Baltimore). 2017;96(22):e7022. doi:10.1097/MD.0000000000007022.

5. Kaur R, Kaur M, Singh J. Endothelial dysfunction and platelet hyperactivity in type 2 diabetes mellitus: molecular insights and therapeutic strategies. Cardiovasc Diabetol. 2018;17(1):121. doi:10.1186/s12933-018-0763-3.

6. Mangano DT; Multicenter Study of Perioperative Ischemia Research Group. Aspirin and mortality from coronary bypass surgery. N Engl J Med. 2002;347(17):1309-17. doi:10.1056/NEJMoa020798.

7. Hillis LD, Smith PK, Anderson JL, Bittl JA, Bridges CR, Byrne JG, et al. 2011 ACCF/AHA guideline for coronary artery bypass graft surgery. A report of the American college of cardiology foundation/American heart association task force on practice guidelines. Developed in collaboration with the American association for thoracic surgery, society of cardiovascular anesthesiologists, and society of thoracic surgeons. J Am Coll Cardiol. 2011;58(24):e123-210. doi:10.1016/j.jacc.2011.08.009.

8. Sousa-Uva M, Head SJ, Milojevic M, Collet JP, Landoni G, Castella M, et al. 2017 EACTS guidelines on perioperative medication in adult cardiac surgery. Eur J Cardiothorac Surg. 2018;53(1):5-33. doi:10.1093/ejcts/ezx314.

9. Ferraris VA, Saha SP, Oestreich JH, Song HK, Rosengart T, Reece TB, et al. 2012 update to the society of thoracic surgeons guideline on use of antiplatelet drugs in patients having cardiac and noncardiac operations. Ann Thorac Surg. 2012;94(5):1761-81. doi:10.1016/j.athoracsur.2012.07.086.

10. Aboul-Hassan SS, StankowskiT, Marczak J, Peksa M, Nawotka M, Stanislawski $\mathrm{R}$, et al. The use of preoperative aspirin in cardiac surgery: a systematic review and meta-analysis. J Card Surg. 2017;32(12):758-74. doi:10.1111/jocs.13250.

11. Zisman E, Erport A, Kohanovsky E, Ballagulah M, Cassel A, Quitt M, et al. Platelet function recovery after cessation of aspirin: preliminary study of volunteers and surgical patients. Eur J Anaesthesiol. 2010;27(7):617-23. doi:10.1097/EJA.0b013e328335b354.

12. Lee J, Kim JK, Kim JH, Dunuu T, Park SH, Park SJ, et al. Recovery time of platelet function after aspirin withdrawal. Curr Ther Res Clin Exp. 2014;76:2631. doi:10.1016/j.curtheres.2014.02.002.

13. Deng Y, Pisklak PV, LeeW, Tolpin DA, Collard CD, Elayda MA, et al. Association between preoperative aspirin-dosing strategy and mortality after coronary artery bypass graft surgery. Ann Surg. 2015;262(6):1150-6.

14. Aboul-Hassan SS, Stankowski T, Marczak J, Peksa M, Nawotka M, Stanislawski $\mathrm{R}$, et al. Timing strategy of preoperative aspirin and its impact on early outcomes in patients undergoing coronary artery bypass grafting: a propensity score matching analysis. J Surg Res. 2020;246:251-9. doi:10.1016/j. jss.2019.09.026.

15. Aboul-Hassan SS, Marczak J, Stankowski T, Peksa M, Nawotka M, Stanislawski $\mathrm{R}$, et al. Association between preoperative aspirin and acute kidney injury following coronary artery bypass grafting. J Thorac Cardiovasc Surg. 2020;160(3):712-9. doi:10.1016/j.jtcvs.2019.08.119.

16. Vandenbroucke JP, von Elm E, Altman DG, Gøtzsche PC, Mulrow CD, PocockSJ, et al. Strengthening the reporting of observational studies in epidemiology (STROBE): explanation and elaboration. Int J Surg. 2014;12(12):1500-24. doi:10.1016/j.jijsu.2014.07.014.

17. Thygesen K, Alpert JS, Jaffe AS, Simoons ML, Chaitman BR, White HD, et al. Third universal definition of myocardial infarction. J Am Coll Cardiol. 2012;60(16):1581-98. doi:10.1016/j.jacc.2012.08.001.

18. Benedetto U, Head SJ, Angelini GD, Blackstone EH. Statistical primer: propensity score matching and its alternatives. Eur J Cardiothorac Surg. 2018;53(6):1112-7. doi:10.1093/ejcts/ezy167.

19. Austin PC. Optimal caliper widths for propensity-score matching when 
estimating differences in means and differences in proportions in observational studies. Pharm Stat. 2011;10(2):150-61. doi:10.1002/pst.433.

20. Dhananjayan R, Koundinya KS, Malati T, Kutala VK. Endothelial dysfunction in type 2 diabetes mellitus. Indian J Clin Biochem. 2016;31(4):372-9. doi:10.1007/ s12291-015-0516-y.

21. Du X, Edelstein D, Obici S, Higham N, Zou MH, Brownlee M. Insulin resistance reduces arterial prostacyclin synthase and eNOS activities by increasing endothelial fatty acid oxidation. J Clin Invest. 2006;1 16(4):107180. doi:10.1172/JCl23354.

22. Giacco F, Brownlee M. Oxidative stress and diabetic complications. Circ Res. 2010;107(9):1058-70. doi:10.1161/CIRCRESAHA.110.223545.

23. Levy JH, Tanaka KA. Inflammatory response to cardiopulmonary bypass. Ann Thorac Surg. 2003;75(2):S715-20. doi:10.1016/s0003-4975(02)04701-x

24. Ballotta A, Saleh HZ, El Baghdady HW, Gomaa M, Belloli F, Kandil H, et al. Comparison of early platelet activation in patients undergoing on-pump versus off-pump coronary artery bypass surgery. J Thorac Cardiovasc Surg. 2007;134(1):132-8. doi:10.1016/j.jtcvs.2007.01.055

25. Møller $\mathrm{CH}$, Steinbrüchel DA. Platelet function after coronary artery bypass grafting: is there a procoagulant activity after off-pump compared with on-pump surgery? Scand Cardiovasc J. 2003;37(3):149-53. doi:10.1080/14017430310001456.

26. Quigley RL, Fried DW, Pym J, Highbloom RY. Off-pump coronary artery bypass surgery may produce a hypercoagulable patient. Heart Surg Forum. 2003;6(2):94-8. doi:10.1532/hsf.698.

27. ASCEND Study Collaborative Group, Bowman L, Mafham M, Wallendszus K, Stevens $W$, Buck $G$, et al. Effects of aspirin for primary prevention in persons with diabetes mellitus. N Engl J Med. 2018:379(16):1529-39. doi:10.1056/ NEJMoa1804988.

28. Deja MA, Kargul T, Domaradzki W, Stącel T, Mazur W, Wojakowski W, et al. Effects of preoperative aspirin in coronary artery bypass grafting: a double-blind, placebo-controlled, randomized trial. J Thorac Cardiovasc Surg. 2012;144(1):204-9. doi:10.1016/j.jtcvs.2012.04.004.

29. Xiao F, Wu H, Sun H, Pan S, Xu J, Song Y. Effect of preoperatively continued aspirin use on early and mid-term outcomes in off-pump coronary bypass surgery: a propensity score-matched study of 1418 patients. PLoS One. 2015;10(2):e0116311. doi:10.1371/journal.pone.0116311. 